\title{
Prácticas parentales de alimentación, autoeficacia y actitudes maternas con niños/as preescolares
}

\section{sonorenses}

\section{Parental Feeding Practices, Self-efficacy and Maternal Attitudes with Sonoran Preschool Children}

Nora Hemi Campos Rivera1; Raúl José Alcázar Olán”; María de Guadalupe Jocobi Zuñiga²; Raquel García Flores²

1 Universidad Iberoamericana de Puebla

2 Instituto Tecnológico de Sonora

\section{Resumen}

El objetivo del estudio fue analizar la manera en que se relacionan las actitudes hacia el sobrepeso y la obesidad en niños, la autoeficacia materna y las prácticas parentales de alimentación. Participaron 300 madres de familia con al menos un/a niño/a de 3 a 7 años $\left(M_{\text {edad }}=5.43, D E=6.1\right)$ de primer a tercer grado a nivel preescolar, con un total de once jardines de niños de una ciudad del noroeste de México. Las madres tenían de 20 a 50 años (Medad $=30.99, D E$ = 6.1). Se utilizó el cuestionario de actitudes, creencias y prácticas de alimentación, la escala de autoeficacia y la escala de actitudes maternas hacia el sobrepeso y la obesidad infantil. Se encontró que el peso percibido del niño está asociado con el peso percibido de la madre, las actitudes hacia el exceso de peso se encuentran relacionadas con las prácticas parentales de alimentación. La autoeficacia tuvo correlaciones significativas con las prácticas parentales de alimentación.

Palabras clave: prácticas parentales, autoeficacia, actitudes, madres, preescolares

1 Nora Hemi Campos Rivera Departamento de Ciencias de la Salud, Universidad Iberoamericana, Campus Puebla. https://orcid.org/0000-0001-9208-0631

1 Raúl José Alcázar Olán Departamento de Ciencias de la Salud, Universidad Iberoamericana, Campus Puebla. https://orcid.org/0000-0001-9439-6293

2 María de Guadalupe Jocobi Zuñiga Departamento de Psicología, Instituto Tecnológico de Sonora. https://orcid.org/0000-0002-1126-9369

2 Raquel García Flores Departamento de Psicología, Instituto Tecnológico de Sonora.

https://orcid.org/0000-0002-2786-5069

Autor para correspondencia: norahemi.campos@iberopuebla.mx 


\begin{abstract}
The objective of this study was to analyze the relationship between attitudes, maternal self-efficacy, and parental feeding practices as it relates to overweight and obese children. Three hundred mothers with at least one child between 3 and 7 years of age $(M a g e=5.43, S D=6.1)$ attending first to third grade at the elementary level, from among eleven kindergartens in a northwestern Mexican city were included in the study. The age of the Mothers ranged between 20 and 50 years (Mage $=30.99, S D=6.1$ ). For this study, we used The Attitudes, Beliefs and Feeding Practices Questionnaire, the Self-Efficacy Scale and the Scale of Maternal Attitudes towards Childhood Overweight and Obesity. We found that the perceived child's weight is associated with the perceived mother's weight; attitudes towards excess weight are linked to parental feeding practices.
\end{abstract}

Key words: parenting practices; self-efficacy; attitudes; mothers; preschoolers

DOI https://doi.org/10.36793/psicumex.v11i1.352

Recibido 25 de abril de 2020

Aceptado 13 de abril de 2021

Publicado 2 de junio de 2021 


\section{Introducción}

La obesidad ha alcanzado proporciones epidémicas a nivel mundial, y cada año mueren como mínimo 2.8 millones de personas a causa de enfermedades relacionadas con la obesidad y el sobrepeso (OMS, 2018). En México, la prevalencia combinada de sobrepeso y obesidad en la población de 5 a 11 años disminuyó ligeramente del $34.4 \%$ en 2012 al $33.2 \%$ en el 2016 (Instituto Nacional de Salud Pública, [INSP], 2016). Sin embargo, en la última encuesta de la ENSANUT, (INSP, 2018) la prevalencia incrementó al $35.6 \%$ (18.1\% para sobrepeso y $17.5 \%$ para obesidad), principalmente en zonas urbanas con un $37.9 \%$, y un 29.7 $\%$ para las rurales.

El estado de Sonora en México se encuentra situado en el noroeste del país, colinda al norte con los Estados Unidos de América a lo largo de una frontera con los estados de Arizona y Nuevo México, al este con el estado de Chihuahua y al sur con el estado de Sinaloa. Los resultados de la ENSANUT 2018 muestran una prevalencia del $73.7 \%$ de sobrepeso y obesidad en la población sonorense mayor a los 20 años, esta cifra es superior por 2 puntos porcentuales a la media nacional y ubica al estado en el octavo lugar.

El comportamiento es similar en los menores de 5 años en donde la prevalencia de sobrepeso y obesidad fue de $14.1 \%$, con una distribución para las localidades urbanas de $14.7 \%$ y para las rurales de 8.7 \%. En el caso de niños en edad preescolar y escolar, son los padres quienes tienen un papel primordial en la prevención del exceso de peso, principalmente en fomentar hábitos y preferencias de alimentación saludables (Birch, 1999). La mayoría de los estudios están enfocados a las madres, pues son ellas quienes pasan más tiempo con los niños y/o son las encargadas de la alimentación, aunque en diversos estudios han involucrado a ambos padres (Noor et al., 2012; Smolak et al., 1997).

Las prácticas de alimentación se refieren a las conductas o comportamientos específicos que caracterizan a las interacciones durante la alimentación de los hijos, un ejemplo de esto puede ser el número de bocados que se ofrecen, la reacción de los padres frente a la aceptación o el rechazo del niño hacia algún 
alimento. La forma en la que se evalúa dicha interacción durante la alimentación se centra en las practicas que se llevan a cabo, por ejemplo, las acciones cuando un niño se niega a comer o de qué forma se prohíben los alimentos (Black y Creed-Kanashiro, 2012). La clasificación de las prácticas de alimentación es diversa, hasta el momento existen algunas con respecto al nombre y la función de cada práctica. En la Tabla 1 se detallan las características de algunas de ellas.

\section{Tabla 1}

Principales prácticas de alimentación parental

\begin{tabular}{lll}
\hline & Presión / & -Se busca que el niño se termine lo que tiene en su \\
estímulo para & plato \\
comer & -Negociaciones (regateo) con el niño \\
Control & -Persuasión para que el niño termine ciertos \\
& & alimentos \\
& Restricción &
\end{tabular}

-Uso de restricción abierta/encubierta

Manipulación

Modelamiento

-Usa la comida como recompensa

-Imponer castigos al no comer

-Muestras de consumo por parte de los padres

con aceptación. 


\begin{tabular}{lll}
\hline & Cambiando y & -Añadir azúcar, mantequilla y salsas \\
Sustitución & modificando & alimento preferido \\
alimentos & -Ocultar alimentos dentro de uno preferido \\
& -Ofrecer alimentos en diversas formas \\
& & -Ofrecer un alimento "más saludable" cuando el \\
& & \\
& niño pide uno "menos saludable"
\end{tabular}

Evitar

-Dar lo que los niños demandan

problemas

-No desalentar hacia algún alimento

-No presentar o fomentar algún alimento nuevo

Razonamiento

-Brindar explicaciones para persuadir a cambiar su comportamiento.

Monitoreo

-Monitorear qué y cuando está comiendo.

-Supervisar y cuantificar lo que come.

Nota. Adaptado de "Parental feeding practices and their relation to child eating behavior: Problems for explanation", de M. L. González-Torres, C. N. Esqueda y M. A. Vaclo, 2018, Revista Mexicana de Trastornos Alimentarios 9(1), pp. 129-142 (http://doi.org/10.22201/fesi.20071523e.2018.1.450); adaptado de "Feeding practices and styles used by a diverse sample of low-income parents of preschool-age children", de Ventura, J. Gromis y Z. Lohse, 2010, Journal of Nutrition Education and Behavior, 42(4), pp. 242-249 (https://doi.org/10.1016/j.jneb.2009.06.002A., 2010).

Las prácticas de alimentación de acuerdo con diversos autores se implementan por diversas razones, por ejemplo, la presión y la restricción para comer están asociadas a la alimentación saludable y medidas 
objetivas de composición corporal en la niñez preescolar (Rodríguez y Ramírez, 2017). En algunos estudios en donde se ha trabajado la alfabetización en salud para padres con hijos/as en edad preescolar, se ha encontrado que está asociada a niveles bajos de autoeficacia parental (Fong et al., 2018).

La autoeficacia, definida como la creencia para llevar a cabo una conducta, funge como promotora de la ingesta dietética protectora contra la obesidad en niños pequeños, cambia durante un período de dos años y medio en una dirección mayoritariamente descendente (Walsh et al., 2019). Se ha documentado que las madres que tienen un sentido firme de autoeficacia suelen disponer de más recursos para potenciar las competencias del hijo; la autoeficacia materna se ha asociado con la capacidad de proveer adaptación, estimulación y alimentar el entorno de crianza de los hijos (Flores et al., 2015).

En pacientes con sobrepeso y obesidad se ha encontrado que entre mayores son los niveles de ansiedad, menor la autoeficacia para bajar de peso y llevar a cabo cada una de las actividades que ayudarían a lograr dicho fin, tales como controlar la ingesta de alimentos, iniciar/mantener la actividad física y regular la compra de alimentos. En cuanto a su relación con la depresión, se encontró que, a mayor depresión, menor fue la autoeficacia para controlar la ingesta de alimentos y para regular su compra (Silva et al., 2019). Por otro lado, la autoeficacia parental está asociada al apoyo social, es decir, la disposición de familiares y amigos en la crianza de sus hijos/as (Izzo et al., 2000).

El comportamiento materno tiene influencia sobre el comportamiento de salud de los niños, es decir, las conductas y actitudes relacionadas con la salud materna con respecto a las prácticas de alimentación y la autoeficacia se vinculan con las conductas relacionadas con la salud de los niños, incluidas las conductas alimentarias, de actividad física y sedentarismo (Chen et al., 2017). La autoeficacia promueve conductas saludables en los primeros años de vida de los niños: propicia la limitación de los alimentos/bebidas no esenciales, la exposición al tiempo de pantalla, la ingesta de frutas y el consumo de agua en niños preescolares (Campbell et al., 2010). 
Además de la autoeficacia, están las actitudes, definidas como una tendencia psicológica que es expresada a través de la evaluación de una entidad en particular con algún grado a favor o en contra (Eagly y Chaiken, 1993). Las actitudes negativas hacia la obesidad han sido asociadas con la creencia incorrecta acerca de las causas del aumento de peso y las responsabilidades del sobrepeso por las personas y sus familiares (Jiménez-Cruz et al., 2008).

Entender las actitudes de los padres, en nuestro caso en las madres, radica en que son ellas quienes toman decisiones en relación con cómo y de qué manera alimentarán a sus hijos/as. Conocer el tipo de actitud hacia la obesidad podría proporcionar información necesaria para el diseño de intervenciones que estén relacionadas a las prácticas y estilos de alimentación hacia sus hijos (Pettigrew et al., 2015).

Los padres deciden qué tipo de práctica utilizan dependiendo del peso que tienen sus hijos/as, y este, a su vez, puede ser diferente comparado con el de los/as hermanos/as. Por ejemplo, en familias con hermanos con peso diferente, los padres informaron que los hermanos con sobrepeso disfrutan de los alimentos y tienen menores niveles de saciedad con respecto a los alimentos en comparación con los hermanos de peso saludable. Los padres utilizan prácticas de alimentación más restrictivas con los/as hijos/as con sobrepeso en comparación con los de peso saludable, y presión para comer y/o prácticas de alimentación con estímulos para comer con los hermanos que tenían un peso saludable en comparación con los hermanos con sobrepeso (Berge et al., 2016).

Sin embargo, el que las madres se enfoquen en el tipo de práctica dependiendo del peso de sus hijos es contraproducente, pues se ha encontrado que las madres de hijos preescolares con sobrepeso y obesidad no perciben que su hijo tiene esta condición; ejercen un estilo de alimentación "no involucrado o indiferente" y en niños con peso normal ejercen un estilo autoritario, que ambos son denominados estilos de crianza en la alimentación (Flores-Peña et al., 2017). 
Niños preescolares con sobrepeso y obesidad que presentan bajo nivel de actividad física, principalmente las niñas, padres con nivel socioeconómico bajo y sin estudios tienden a tener un nivel nutricional precario (Latorre et al., 2016). Las prácticas de alimentación son parte de una cultura y tradición, varían dependiendo del grupo y características del lugar, la disponibilidad de los alimentos, aceptación o rechazo por parte de los niños y de las creencias que se tenga hacia ciertos alimentos. Intentar cambiar estas prácticas requeriría de tiempo, el cambio de creencias, actitudes y que este se perciba como una amenaza para la salud (Birch, 2006).

Por otro lado, estudios epidemiológicos demuestran una fuerte asociación entre obesidad materna e infantil; sugieren que una predisposición a la ganancia de peso podría ser influenciada por experiencias prenatales. Las influencias del desarrollo sobre la obesidad en los niños quienes han nacido de madres obesas son en parte la consecuencia, a largo plazo, de los efectos de la obesidad materna en calidad durante la vida fetal (Robinson, 2011).

Así mismo, el tipo de alimentación está asociada con el índice de masa corporal (IMC) de padres e hijos. Se han reportado diferencias en el consumo de alimentos como frutas y verduras entre niños y padres con peso normal y sobrepeso. Los niños de peso normal consumen con más frecuencia frutas y verduras que los niños con sobrepeso.

De igual manera las madres con un hijo con sobrepeso consumen con menor frecuencia frutas y verduras (Vanhala et al., 2011). Mientras tanto, se sabe que niños de familias obesas suelen sobrealimentarse en respuesta a indicadores emocionales. Jahnke y Warschburger (2008) mencionan que la trasmisión familiar de conductas de alimentación está relacionada con el peso como un factor esencial para el inicio de la obesidad, consideran que conductas externas de alimentación como la sensibilidad a la comida o velocidad al comer están asociadas con la alimentación emocional en relación con personas con peso normal. 
Son diversas las variables que están asociadas al sobrepeso y la obesidad en los niños, sin embargo y de acuerdo con la revisión realizada, variables como las actitudes, prácticas de alimentación y la autoeficacia están interrelacionadas. Por lo tanto, el objetivo de este estudio fue analizar de qué manera se relacionan las actitudes hacia el sobrepeso y la obesidad en niños, la autoeficacia hacia el cuidado del peso del/a niño/a y las prácticas parentales de alimentación de madres con hijos/as en edad preescolar.

\section{Metodología}

\section{Participantes}

Se seleccionaron de forma no probabilística 300 madres de familia con al menos un niño en edad de 3 a 7 años $\left(M_{\text {edad }}=5.43, D E=6.1\right)$ de primer a tercer grado a nivel preescolar, con un total de once jardines de niños de una ciudad del noroeste de México. La edad de las madres osciló entre 20 y 50 años $\left(M_{e d a d}=\right.$ 30.99, $D E=.82)$; el $22.3 \%$ eran solteras, el $75.3 \%$ casadas y el $2.3 \%$ no especificó. La escolaridad de las participantes indicó en primaria $4.3 \%$, secundaria $22.7 \%$, preparatoria $31.3 \%$, licenciatura $27.3 \%$, posgrado $1.7 \%$ y carrera técnica $5.3 \%$. Las ocupaciones de las madres fueron actividades en el hogar/ama de casa $52.7 \%$, negocio propio $4.7 \%$, empleadas $21 \%$, profesionistas $6.3 \%$, estudiantes $2.3 \%$, oficio 2.3 $\%$ y técnico $3 \%$.

\section{Instrumentos}

\section{Prácticas parentales de alimentación}

Adaptación mexicana del Child Feeding Questionnaire (CFQ) (Birch, Fisher, Grimm-Thomas, Markey, y Sawyer, 2001), adaptada por Navarro (2016). Compuesto por 33 reactivos $(\alpha=.858)$, se compone de 8 factores que explican el $59.99 \%$ de la varianza: a) peso percibido del niño $(\alpha=.834)$, b) monitoreo ( $\alpha$ $=.866)$, c) presión para comer $(\alpha=.720), \mathrm{d})$ peso percibido de la madre $(\alpha=.677)$, e) restricción $(\alpha=$ $.757), \mathrm{f})$ restricción como método de disciplina $(\alpha=.786)$, g) preocupación por el peso del niño $(\alpha=.683)$ y h) responsabilidad percibida $(\alpha=.652)$. 


\section{Escala de autoeficacia materna para la prevención del exceso de peso (Campos et al., 2018)}

Conformado por 11 reactivos, es una escala unidimensional $(\alpha=.90$, p. ej., tomar las mejores decisiones respecto al cuidado de peso de mi hijo), con una varianza explicada de $52 \%$. Todos los ítems se contestaron en una escala tipo Likert con cinco opciones de respuesta desde 1 (nada capaz) hasta 5 (totalmente capaz).

\section{Escala de actitudes hacia el exceso de peso (Campos y Sotelo, 2019)}

Compuesto por 10 reactivos, conformada por dos dimensiones: a) negativa $(\alpha=.81$, p. ej., es preocupante de los niños suban de peso) con una varianza explicada de $40.3 \%$, y b) consecuencias $(\alpha=.73$, p. ej., el sobrepeso te hace sentir depresión), varianza explicada de $5.7 \%$. La escala total tiene un $\alpha=.85 \mathrm{y}$ una varianza que explica el $46.1 \%$. Todos los ítems se puntúan en una escala de cinco opciones de respuesta, desde 1 (totalmente en desacuerdo) hasta 5 (totalmente de acuerdo).

\section{Procedimiento}

Se solicitó autorización a las autoridades de las instituciones educativas de nivel preescolar del sector público, además de pedir el permiso correspondiente para la aplicación de los instrumentos a las madres de familia, garantizando la confidencialidad de la información y su consentimiento informado. Las madres recibieron los instrumentos aplicados por medio de sus hijos, debido a la falta de tiempo y que la mayoría se encontraba laborando; una vez finalizada la recolección de instrumentos se procedió a la captura de información mediante el programa Statiscal Package for the Social Sciences (SPSS) versión 21 y, posteriormente, se realizaron los análisis de los datos.

\section{Resultados}

Con la intención de conocer de qué manera se relacionan las prácticas de alimentación parental, la autoeficacia materna hacia el cuidado del peso del hijo y las actitudes hacia el exceso de peso, se trabajó con 
madres de 20 hasta 50 años $(\bar{x}=30.99, D E=6.108)$ con un hijo/a en edad preescolar, con distintas escolaridades tales como preparatoria $(31.3 \%)$, licenciatura $(27 \%)$, secundaria $(22.7 \%)$, carrera técnica $(5.3 \%)$, primaria $(4.3 \%)$ y posgrado $(1.7 \%)$. Por otro lado, las participantes indicaron su tipo de ocupación, hogar $(52.7 \%)$, empleada $(21 \%)$, profesionista $(6.3 \%)$, negocio propio $(4.3 \%)$, puesto técnico (3\%), estudiantes $(2.3 \%)$ y otros oficios $(2.3 \%)$. El $22.3 \%$ de las madres reportaron ser solteras y el $75.3 \%$ casadas, mientras que el $2.3 \%$ no especificó.

Con base en los resultados el $69.7 \%$ de la muestra indicó que el hijo de la participante no practicaba deporte, mientras que el $20.7 \%$ sí lo practicaba, el tiempo que le dedicaban a la semana fue de 3 horas (5 $\%)$. El deporte más practicado fue el futbol (8.3\%), seguido de bicicleta (2.3\%) y baile/ballet (2. \%) mientras que la actividad que menos se practicaba fue jugar con una pelota $(0.3 \%)$ y usar la patineta $(0.3 \%)$.

Los análisis de los resultados mostraron que las madres utilizaban más el monitoreo con una $\bar{x}=4.37$ y una $D E=.78$, lo que significa que supervisan y cuantifican lo que comen sus hijos, y manifiestan una alta responsabilidad percibida $(\bar{x}=4.16$ y una $D E=.64)$ con relación a la alimentación y cuidado del peso de sus hijos/as, además de la preocupación por el peso del hijo/a $(\bar{x}=4.16$ y una $D E=.994)$. Es importante destacar que la media más elevada fue la de autoeficacia $(\bar{x}=4.46$ y una $D E=.612)$, es decir, las madres expresaban su creencia sobre la capacidad para prevenir el exceso de peso de sus hijos/as.

Con respecto a la percepción que tienen del peso de sus hijos, se obtuvo una $\bar{x}=2.17$ y una $D E=.73$, lo que significa que las madres perciben el peso de sus hijos como normal. Posteriormente obtuvimos una $\bar{x}$ $=3.61$ y una $D E=.91$ en la dimensión presión para comer, lo que significa que las madres llevan a cabo esta práctica, es decir, que las madres buscan que los niños/as se terminen lo que tienen en su plato.

Así mismo, con respecto a las actitudes hacia el exceso de peso obtuvimos una $\bar{x}=3.37$ y una $D E=$ .54 de la escala total, en la dimensión negativa obtuvimos una media de $\bar{x}=3.44$ y una $D E=.55$ y en la 
dimensión de consecuencias se observa una $\bar{x}=3.26$ y una $D E=.65$, lo que significa que manifiestan actitudes negativas hacia el exceso de peso.

Por otro lado, con respecto a la percepción del peso de las madres obtuvimos una $\bar{x}=3.03 \mathrm{y}$ una $D E=.74$, lo que significa que se perciben ligeramente pasadas de peso, pero es muy poca la diferencia comparada con la percepción que tienen con sus hijos/as. En cuanto a la restricción como disciplina $\bar{x}=3.03$ y una $D S=1.135$, y la preocupación por el peso del niño una $\bar{x}=4.16$ y $D E=.994$ (ver Tabla 1$)$.

\section{Tabla 1}

Medias y desviaciones estándar de las variables

\begin{tabular}{lcc}
\hline & $\bar{x}$ & DE \\
\hline Prácticas & $\mathbf{3 . 4 0}$ & $\mathbf{. 5 3 0}$ \\
Peso percibido niño & 2.17 & .737 \\
Monitoreo & 4.37 & .787 \\
Presión para comer & 3.61 & .916 \\
Peso percibido madre & 3.03 & .746 \\
Restricción & 3.99 & .915 \\
Restricción como disciplina & 3.03 & 1.135 \\
Preocupación por el peso del & 4.16 & .994 \\
niño & 4.16 & .642 \\
Responsabilidad percibida & $\mathbf{3 . 3 7}$ & $\mathbf{. 5 4 7}$ \\
Actitudes & 3.44 & .554 \\
Negativa & 3.26 & .653 \\
Consecuencias & $\mathbf{4 . 4 6}$ & $\mathbf{. 6 1 2}$ \\
Autoeficacia & $\mathbf{2 5 . 2 4}$ & $\mathbf{8 . 4 7}$ \\
\hline IMC de la Madre &
\end{tabular}

En otro contexto, respecto al autorreporte del índice de masa corporal de las madres obtuvimos una $\bar{x}=25$ y una $D E=8.47$, de acuerdo con la OMS, lo que significa que la mayoría de las mamás reportan que tienen sobrepeso, tomando en cuenta que mayor o igual a 25 se clasifica como sobrepeso (ver Figura 1).

\section{Figura 1}

Porcentaje del IMC autorreportado por las madres 


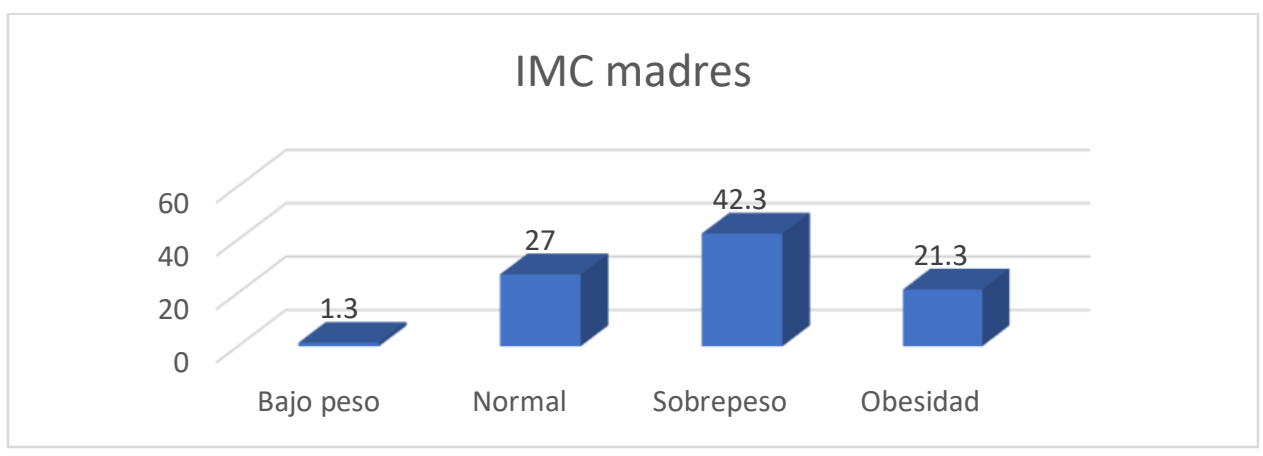

A continuación, en la Tabla 3 se muestra la matriz de correlaciones de las variables de estudio, en general las correlaciones son muy bajas y medias. Las actitudes negativas tienen una asociación lineal baja con la preocupación para comer $(r=.18, p<0.01)$, al igual que con la restricción $(r=.17, p<0.01)$ y presión para comer $(r=.19, p<0.01)$. Con respecto a la edad del niño solo se encontró asociación lineal baja con respecto a las actitudes que tienen que ver con las consecuencias hacia la obesidad infantil $(r=.16, p<0.01)$. Con respecto a la práctica de alimentación responsabilidad se encontró asociación lineal baja con restricción $(r=.16, p<0.01), \mathrm{y}$ asociación lineal media con monitoreo $(r \mathrm{P}=.38, p<0.01)$ y la autoeficacia $(r \mathrm{P}=.30, p$ $<0.01)$.

Por otro lado, la preocupación por el peso del niño tiene una asociación lineal media con restricción $(r=.31, p<0.01)$, baja con restricción como disciplina $(r=.26, p<0.01)$, monitoreo $(r=.20, p<0.01) \mathrm{y}$ actitudes como consecuencias $(r=.20, p<0.01)$, finalmente, con una asociación lineal baja con la autoeficacia $(r=.14, p<0.01)$.

La práctica de alimentación restricción tiene una asociación lineal baja con restricción como disciplina $(r=.27, p<0.01)$, presión para comer $(r=.20, p<0.01)$ y autoeficacia $(r=.26, p<0.01)$, además una asociación lineal media con monitoreo $(r=.39, p<0.01)$.

Con respecto a la práctica restricción como disciplina se obtuvieron asociaciones lineales bajas con el monitoreo $(r=.21, p<0.01)$, presión para comer $(r=.20, p<0.01)$, actitudes como consecuencias $(r=.20$, $p<0.01)$, peso percibido del niño $(r=.15, p<0.01)$ y autoeficacia $(r=.18, p<0.01)$. El IMC de la madre 
tuvo una asociación lineal baja con la práctica presión para comer $(r=.17, p<0.01)$, no se encontraron más asociaciones con esta variable. Con respecto a la práctica monitoreo tuvo una asociación lineal baja con presión para comer $(r=.20, p<0.01)$. Las actitudes negativas como consecuencias tuvieron una asociación lineal baja con presión para comer $(r=.25, p<0.01)$ y autoeficacia $(r=.16, p<0.01)$. Finalmente, se encontró que el peso percibido hacia el niño tuvo una asociación lineal media con el peso percibido de la madre $(r=.33, p<0.01$.$) .$

\section{Tabla 3}

Matriz de correlaciones con las variables de estudio

\begin{tabular}{|c|c|c|c|c|c|c|c|c|c|c|c|c|c|c|}
\hline & & 1 & 2 & 3 & 4 & 5 & 6 & 7 & 8 & 9 & 10 & 11 & 12 & 13 \\
\hline 1 & $\begin{array}{l}\text { Negativa } \\
\text { (actitudes) }\end{array}$ & - & & & & & & & & & & & & \\
\hline 2 & Edad niño & .050 & - & & & & & & & & & & & \\
\hline 3 & $\begin{array}{l}\text { Responsabilidad de } \\
\text { los padres }\end{array}$ & .110 & -.044 & - & & & & & & & & & & \\
\hline 4 & Preocupación & $.185^{* *}$ & .033 & -.003 & - & & & & & & & & & \\
\hline 5 & Restricción & $.179^{* *}$ & -.051 & $.162^{* *}$ & $.310^{* *}$ & - & & & & & & & & \\
\hline 6 & $\begin{array}{l}\text { Restricción como } \\
\text { disciplina }\end{array}$ & $.164^{*}$ & .077 & .081 & $.269^{* *}$ & $.278^{* *}$ & - & & & & & & & \\
\hline 7 & IMC madre & .034 & .097 & .022 & .013 & .022 & .076 & - & & & & & & \\
\hline 8 & Monitoreo & .078 & -.023 & $.385^{* *}$ & $.208^{* *}$ & $.395^{* *}$ & $.217^{* *}$ & .075 & - & & & & & \\
\hline 9 & Presión para comer & $.195^{* *}$ & .090 & .108 & $.125^{*}$ & $.232^{* * *}$ & $.315^{* *}$ & $.178^{* *}$ & $.205^{* *}$ & - & & & & \\
\hline 10 & $\begin{array}{l}\text { Consecuencias } \\
\text { (actitudes) }\end{array}$ & $.697^{* *}$ & $.163^{* * *}$ & .089 & $.206^{* *}$ & .117 & $.208^{* *}$ & .035 & .112 & $.252^{* *}$ & - & & & \\
\hline 11 & $\begin{array}{l}\text { Peso percibido } \\
\text { madre }\end{array}$ & -.020 & -.059 & .052 & .012 & .112 & -.015 & $.155^{* *}$ & .002 & .048 & -.014 & - & & \\
\hline 12 & $\begin{array}{l}\text { Peso percibido } \\
\text { niño }\end{array}$ & -.049 & $.143^{*}$ & -.030 & .079 & .010 & $.153^{* *}$ & .059 & -.016 & $.132^{*}$ & .027 & $.335^{* *}$ & - & \\
\hline 13 & Autoeficacia & .108 & -.066 & $.301^{* *}$ & $.170^{* *}$ & $.267^{* *}$ & $.186^{* * *}$ & -.041 & $.378^{* *}$ & $.163^{* *}$ & .047 & -.079 & -.039 & - \\
\hline
\end{tabular}

$*$ Sig. $<.05 * *$ Sig. $<.01 p=.10$ bajo, .30 media, .50 alta.

\section{Discusión}

De acuerdo con el objetivo de la investigación podemos considerar que nuestras variables de estudio tienen poca relación entre sí, las correlaciones obtenidas fueron bajas. Cabe destacar que las relaciones más importantes a pesar de lo bajas fueron las de responsabilidad de los padres con el monitoreo, es decir, los 
padres que se sienten responsables de la alimentación de sus hijos/as tienden a establecer la práctica de alimentación monitoreo (supervisar y cuantificar lo que come) (González-Torres et al., 2018), pero a su vez tiene asociación con la autoeficacia para prevenir el exceso de peso en los niños.

En algunos estudios se ha buscado la asociación entre la percepción de responsabilidad que tienen los padres con la alimentación de sus hijos/as con un pobre o escaso funcionamiento familiar como variable mediadora de problemas de conducta y bajos niveles de prácticas de alimentación como protectores del exceso de peso (percepción de la responsabilidad de los padres y monitoreo de alimentos no saludables); sin embargo, no se ha encontrado asociación alguna (Warnick et al., 2019).

En una revisión sistemática se buscó analizar la relación de los estilos de crianza enfocados a la alimentación y las prácticas parentales de alimentación, sus principales hallazgos fueron que los padres que suelen ser cálidos tienden a utilizar el monitoreo como práctica de alimentación, además de tener una asociación inversa con los padres que utilizan un estilo de crianza permisivo. Aunque en este mismo estudio encontraron que los padres permisivos tienden a utilizar la práctica presión para comer en algunas circunstancias (Collins et al., 2014).

Aunque hay mucha discrepancia en si existe relación entre los estilos de crianza y las prácticas de alimentación, es claro que los padres sí difieren de la práctica dependiendo de cómo sea la relación con sus hijos/as. Sin embargo, en nuestro estudio las cuidadoras primarias fueron las madres, en algunos estudios reportan la importancia de quién es el que está al cuidado de los niños y las niñas, lo que implica que las prácticas de alimentación se modifican o cambian.

Bernadette (2019) encontró que el $47 \%$ de los abuelos, cuando son los cuidadores primarios, rara vez o nunca establecen un límite máximo en la cantidad de alimentos no saludables consumidos. En comparación, los cuidadores primarios (muchas veces las madres) tienden a establecer un límite máximo para la cantidad de alimentos poco saludables que comen sus hijos/as y eligen una amplia variedad de 
alimentos. Estos hallazgos respaldan la necesidad y la importancia que tiene identificar al cuidador primario/a con la intención de sensibilizar cuando no son las madres.

Otra de las variables importantes que influyen en las prácticas de alimentación es el índice de masa corporal (IMC) de las madres o cuidadores/as primarios/as; en nuestros resultados encontramos que el IMC de la madre tuvo una asociación con la práctica presión para comer y la mayoría de las mamás se reportan con sobrepeso. Esto se corrobora en algunos estudios en donde se menciona que las madres con sobrepeso/obesidad que llevan a cabo más control (presión para comer y restricción) sobre la alimentación en sus hijos proporcionan menos equilibrio y variedad alrededor de los alimentos. Reportan un ambiente de comida casera menos saludable y no llevan a cabo modelamiento de alimentación saludable frente a sus hijos. Las madres con sobrepeso/obesidad parecen participar en actividades generalmente menos saludables que las madres con peso saludable, además de que perciben a sus hijos/as más evasivos con la comida, pero no con las bebidas (Haycraft et al., 2017).

Así mismo, encontramos actitudes negativas hacia el exceso de peso y hacia las consecuencias que genera la obesidad infantil. Las actitudes negativas como las consecuencias tuvieron una asociación con presión para comer y la autoeficacia de las madres. Es decir, las madres manifiestan y son conscientes de lo que representa la obesidad.

Por desgracia esto no es suficiente para prevenir el sobrepeso y la obesidad desde casa. En un estudio se encontró que los padres expresan creencias y actitudes positivas sobre la importancia de alimentación saludable para sus hijos pequeños, ellos mismos y sus familias. Sin embargo, la mayoría informó llevar a cabo prácticas familiares como comer fuera y llevar comida comprada, aspectos que se han relacionado con un mayor riesgo de obesidad entre los niños latinos. Los padres están más involucrados en la actividad física de los niños que en su alimentación, aunque varios padres informan que participan predominantemente en 
actividades sedentarias y luchan para establecer límites en el tiempo de pantalla de los niños (Linsay et al., 2017).

Por otro lado, encontramos asociación entre la preocupación por el peso del niño con las prácticas de restricción, restricción como disciplina, monitoreo, la autoeficacia y las actitudes como consecuencias. Estos resultados son consistentes con los encontrados por Swyden et al. (2017), en donde encontraron que los padres preocupados por el peso de sus hijos tienden a utilizar prácticas de alimentación restrictivas, aunque este estudio también encontró asociación entre el estrés materno y la edad de sus hijos. Encontraron que, a mayor edad, los padres tienden a preocuparse más por el peso de sus hijos/as. En nuestro estudio, la edad del niño tuvo asociación con las actitudes respecto a las consecuencias hacia la obesidad infantil.

Con respecto a la autoeficacia, es decir, la creencia que tienen las madres sobre la capacidad de mantener una alimentación saludable y un peso estable, encontramos asociación con la preocupación de los padres. Se ha estudiado esta variable con los problemas conductuales de sus hijos/as. Rocha (2012) identificó una asociación negativa entre estas dos variables, es decir, a menor autoeficacia materna existía más dificultad para manejar los problemas conductuales de sus hijos/as. Con respecto a la autoeficacia y los estilos de crianza se ha encontrado que los padres con niños desadaptados experimentan una autoeficacia baja (Aminayi et al., 2015).

Las prácticas de alimentación que los padres utilizan dependen de múltiples factores. Las llamadas prácticas de alimentación coercitiva, como el uso de presión para comer o el uso de alimentos como recompensas, se deben evitar ya que pueden crear asociaciones negativas con la comida y conducen a rechazos de alimentos. En cambio, los cuidadores pueden modelar comer y disfrutar de la comida. Las recompensas no alimentarias, como elogios o pegatinas, también se pueden usar para alentar a los niños a probar una comida sin resultados negativos (Fries y Van der Horst, 2019). 


\section{Conflicto de intereses}

Los autores declaran no tener conflictos de intereses.

\section{Referencias}

Aminayi, M., Roshan, R., Shairi, M. y Moharreri, F. (2015). Comparative study of parenting styles and parenting self-efficacy in mothers of children with and without anxiety symptoms. Journal of $\begin{array}{lllll}\text { Fundamentals } \quad \text { of } & \text { Mental } & \text { 186-191. }\end{array}$ https://pdfs.semanticscholar.org/3910/e92c26d37ac50049bbb21c68842c4ce04960.pdf?_ga=2.3596445 $1.2062395560 .1587520191-208937994.1587520191$

Berge, J., Tate, A., Trofholz, A., Conger, K. y Neumark-Sztainer, D. (2016). Sibling eating behaviours and parental feeding practices with siblings: similar or different? Public Health Nutrition, 19(13), 24152423. https://doi.org/10.1017/S1368980016000860

Bernadette, Q. M., Jia, M. H., Ka, S. Y., Xueling, S., Miho, A. y Foong-Fong, C. (2019). Feeding-related knowledge, attitudes, and practices among grandparents in Singapore. Nutrients, 11, 1696. https://doi.org/10.3390/nu11071696

Birch, L. (1999). Development of food preferences. Annual Review Nutrition, 19, 41-62.

Birch, L. (2006). Child Feeding Practices and the Etiology of Obesity. Obesity, 14(3), 343-344. https://doi.org/10.1038/oby.2006.45

Black, M. M. y Creed-Kanashiro, H. M. (2012). ¿Cómo alimentar a los niños?: la práctica de conductas alimentarias saludables desde la infancia. Revista Peruana de Medicina Experimental y Salud Pública, 29(3), 373-378. https://www.scielosp.org/article/rpmesp/2012.v29n3/373-378/

Campbell, K., Hesketh, K., Silverii, A. y Abbott, G. (2010). Maternal self-efficacy regarding children's eating and sedentary behaviours in the early years: associations with children's food intake and 
sedentary behaviours. International Journal of Pediatric Obesity,5(6), 501-508. https://doi.org/10.3109/17477161003777425

Campos, N. y Sotelo, T. (2019). Design and validation of a scale of maternal attitudes towards childhood overweight and obesity. Acta Colombiana de Psicología, 22(2), 148-162. https://doi.org/10.14718/ACP.2019.22.2.8

Campos, N., López, G., García, R., Reyes, L., García, C. y Acosta, C. (2018). Diseño y construcción de la Escala de autoeficacia materna para la prevención del exceso de peso. Revista Mexicana de Trastornos Alimentarios, 9(2), 160-169. https://doi.org/10.22201/fesi.20071523e.2018.2.531

Collins, C., Duncanson, K. y Burrows, T. (2014). A systematic review investigating associations between parenting style and child feeding behaviours. Journal of Human Nutrition and Dietetics, 27, $557-$ 568. https://doi.org/10.1111/jhn.12192

Chen, J., Guo, J., Esquivel, J. y Chesla, C. (2017). Like mother, like child: the influences of maternal attitudes and behaviors on weight-related health behaviors in their children. Journal of Transcultural Nursing, 29(6), 523-531. https://doi.org/10.1177/1043659617736883

Eagly, A. y Chaiken, S. (1993). The psychology of attitudes. Thompson.

Instituto Nacional de Salud Pública (2016). Encuesta Nacional de Salud y Nutrición: Resultados Nacionales. http://ensanut.insp.mx/

Instituto Nacional de Salud Pública (2018). Encuesta Nacional de Salud y Nutrición: Resultados Nacionales. http://ensanut.insp.mx/

Flores, Y., Rocha, L., Cárdenas, V. M., Haslam, D., Mejia, A. y Sanders, M. (2015). Conductas del estilo de vida del hijo relacionadas con el peso y autoeficacia materna para manejarlas. Nutrición Hospitalaria, 32(1), 144-150. https://doi.org/10.3305/nh.2015.32.1.8855 
Flores-Peña, Y., Acuña-Blanco, A., Cárdenas-Villarreal, V., Amaro-Hinojosa, M., Pérez-Campa, M. y Elenes-Rodríguez, J. (2017). Asociación de la percepción materna del peso del hijo y estilos maternos de alimentación infantil. Nutrición Hospitalaria, 34(1), 51-58. https://doi.org/10.20960/nh.975

Fong, H., Rothman, E., Garner, A., Ghazarian, S., Morley, D., Singerman, A. y Bair-Merritt, M. (2018). Association between health literacy and parental self-efficacy among parents of newborn children. The Journal of pediatrics, 202, 265-271. https://doi.org/ 10.1016/j.jpeds.2018.06.021

Fries, L. y Van der Horst, K. (2019). Parental Feeding Practices and Associations with Children's Food Acceptance and Picky Eating. Nurturing a Healthy Generation of Children: Research Gaps and Opportunities, 91, 31-39. https://doi.org/10.1159/000493676

González-Torres, M. L., Esqueda, C. N. y Vaclo, M. A. (2018). Parental feeding practices and their relation to child eating behavior: Problems for explanation. Revista Mexicana de Trastornos Alimentarios. 9(1), 129-142. http://doi.org/10.22201/fesi.20071523e.2018.1.450

Haycraft, E., Karasouli, E. y Meyer, C. (2017). Maternal feeding practices and children's eating behaviours: A comparison of mothers with healthy weight versus overweight/obesity. Appetite, 116, 395-400.

Izzo, C., Weiss, L., Shanahan, T. y Rodriguez-Brown, F. (2000). Parental self-efficacy and social support as predictors of parenting practices and children's socioemotional adjustment in Mexican immigrant families. Journal of Prevention $y$ Intervention in the Community,20(1-2), 197-213. https://doi.org/10.1300/J005v20n01_13

Jahnke, D. L. y Warschburger, P. A. (2008). Familial transmission of eating behaviors in preschool-aged children. Obesity, 16(8), 1821-1825. https://doi.org/10.1038/oby.2008.255

Jiménez-Cruz, A., Castellón-Zaragoza, A. M., García-Gallardo, J. L., Bacardí-Gascón, M. y Hovell, M. F. (2008). Strong beliefs on personal responsibilities and negative attitudes towards the child with 
obesity among teachers and parents. Revista Biomédica, 19(2), 84-91. http://www.revbiomed.uady.mx/pdf/rb081922.pdf

Latorre, P., Mora, D. y García, F. (2016). Prácticas de alimentación, actividad física y condición física de niños preescolares españoles: Influencia de variables sociodemográficas. Archivos Argentinos de Pediatría, 114(5), 441-447. https://doi.org/10.5546/aap.2016.441

Linsay, A. C., Wallington, S. F., Muñoz, M. A. y Greaney, M. L. (2017). A qualitative study conducted in the USA exploring Latino fathers' beliefs, attitudes and practices related to their young children's eating, physical activity and sedentary behaviours. Public Health Nutritions, 21(2), 403-415. https://doi./10.1017/S1368980017002579

Navarro, C. G. (2006). Promoción de Estilos de Vida Saludables: El Caso de la Alimentación Infantil [Tesis de doctorado, no publicada]. Facultad de Psicología, Universidad Nacional Autónoma de México.

Navarro, G. (2016). Validación psicométrica de la adaptación mexicana del Child Feeding Questionnaire Psychometric. Acta de investigación psicológica, 6(1), 2337-2349. https://doi.org/10.1016/S20074719(16)30054-0

Noor, A., Leelavathi, M., Shamsul, A., Hizlinda, T., Khairani, O. y Fatimah, A. (2012). Parental concerns and control in feeding of 9 to 12-year-old children in a primary school in Kuala Lumpur, Malaysia. Malaysian Journal Nutrition, 18(1), 47-55.

Organización Mundial de la Salud. (2018). Obesidad y sobrepeso. https://www.who.int/es/news-room/factsheets/detail/obesity-and-overweight

Pettigrew, S., Jongenelis, M., Quester, P., Champman, K. y Miller, C. (2015). Dimensions of parents' attitudes to unhealthy foods and beverages. Food Quality and Preference, 44, 179-182. 10.1016/j.foodqual.2015.05.001 
Rocha, L. (2012). Autoeficacia materna y problemas conductuales del hijo relacionados al peso [Tesis de maestría]. Universidad Autónoma de Nuevo León, Monterrey. http://eprints.uanl.mx/3181/1/1080256548.pdf

Robinson, S. (2011). A Link between Maternal and Childhood Obesity. Global Perspectives on Childhood Obesity (pp. 147-156). Debasis Bagchi.

Rodríguez, G. y Ramírez, N. (2017). Prácticas parentales, alimentación saludable y medidas objetivas de composición corporal en la niñez preescolar. Actualidades en Psicología, 31(122), 61-73. https://doi.org/10.15517/ap.v31i122.26411

Silva, C., Fernández, N. y Rodríguez, N. (2019). Depresión, ansiedad y autoeficacia para bajar de peso en mujeres en tratamiento nutricional. Psicología y Salud,29(1), 41-49. http://psicologiaysalud.uv.mx/index.php/psicysalud/article/view/2567/4462

Smolak, L., Levine, M. y Schermer, F. (1997). Parental input and weight concerns among elementary school children. John Wiley y Sons, 25(3), 263-271. https://doi.org/10.1002/(sici)1098108x(199904)25:3<263:aid-eat3>3.0.co;2-y

Swyden, K., Sisson, S. B., Morris, A.S., Lora, K., Weedn, A. E., Copeland, K. A. y DeGrace, B. (2017). Association between maternal stress, work status, concern about child weight, and restrictive feeding practices in preschool children. Matern Child Health Journal, 21, 1349-1357. https://doi.org/10.1007/s10995-016-2239-y

Vanhala, M. L., Keinänen-Kiukaanniemi, S. M., Kaikkonen, K. M., Laitinen, J. H., y Korpelainen, R. I. (2011). Factors associated with parental recognition of a child's overweight status - a cross sectional study. BMC Public Health, 11(1), 665. https://doi.org/10.1186/1471-2458-11-665 
Ventura, A., Gromis, J. y Gembeck, Z. (2010). Feeding practices and styles used by a diverse sample of lowincome parents of preschool-age children. Journal of Nutrition Education and Behavior, 42(4), 242249. https://doi.org/10.1016/j.jneb.2009.06.002

Walsh, A., Hesketh, K., Hnatiuk, J. y Campbell, K. (2019). Paternal self-efficacy for promoting children’s obesity protective diets and associations with children's dietary intakes. International Journal of Behavioral Nutrition and Physical Activity, 16(1), 2-8. https://doi.org/10.1186/s12966-019-0814-5

Warnick, J. L., Stromberg, S. E., Krietsch, K. M. y Janicke, D.M. (2019). Family functioning mediates the relationship between child behavior problems and parent feeding practices in youth with overweight or obesity. Translational Behavioral Medicine, 9(3), 431-439. https://doi.org/10.1093/tbm/ibz050

Cómo citar este artículo: Campos Rivera, N. H., Alcázar Olán, R. J., Jocobi Zuñiga, M. de G..., \& García Flores, R. (2021). Prácticas parentales de alimentación, autoeficacia y actitudes maternas con niños/as preescolares sonorenses. Psicumex, 11(1), 1-23, e352. https://doi.org/10.36793/psicumex.v11i1.352 